\title{
Managing personal communication systems in a multi-domain environment
}

\author{
A. Richter, M. Tschichholz \\ GMD FOKUS, Hardenbergplatz 2, 10623 Berlin, Germany \\ Phone: +49-30-25499-200 - Fax: +49-30-25499-202 \\ E-Mail: \{richter, tschichholz\}@fokus.gmd.de
}

\begin{abstract}
The ACTS project PROSPECT is investigating the integrated management of a variety of services in a multi-domain tele-education environment. In the project's second trial the focus is on support for mobile students and teachers, enabling them to access and use advanced multimedia services from locations other than their home base. A Personal Communication System (PCS) is being defined that can support mobile user requirements in such a tele-education environment.

Support for mobility has several implications for management. The management systems of multiple organisations need to co-operate to support the provision of tele-educational services to mobile students and teachers with the particular features required by the users. Specific PCS management issues are concerned with user profile management and management of user access to their customised working environment. The question of how to account and charge for resource usage when services can be used in domains other than the user's home domain must be considered, as must the requirements for security when crossing multiple domains. The PCS-specific management functions fall into three different categories. First, providing locality-independent access to a service, second, customising access and use of services from different locations for individual users, and third dealing with charges caused by service usage from external domains. Many of the current architectural concepts available today are not sufficient for modelling the management of personal communication systems in a multi-domain environment. The PCS is therefore enhancing these concepts to provide for the management of student and teacher mobility in the PROSPECT context.
\end{abstract}

